\title{
Society for Translational Medicine expert consensus on the use of antibacterial drugs in thoracic surgery
}

\author{
Shanqing $\mathrm{Li}^{1}$, Zhijun $\mathrm{Han}^{1}$, Jie $\mathrm{He}^{2}$, Shugeng $\mathrm{Gao}^{2}$, Deruo Liu ${ }^{3}$, Lunxu Liu ${ }^{4}$, Jianxing $\mathrm{He}^{5,6}$, Yunchao \\ Huang $^{7}$, Shidong Xu ${ }^{8}$, Weimin Mao ${ }^{9}$, Qunyou Tan $^{10}$, Chun Chen ${ }^{11}$, Xiaofei Li ${ }^{12}$, Zhu Zhang ${ }^{13}$, Gening \\ Jiang $^{14}$, Lin $\mathrm{Xu}^{15}$, Lanjun Zhang ${ }^{16}$, Jianhua $\mathrm{Fu}^{16}$, Hui $\mathrm{Li}^{17}$, Qun Wang ${ }^{18}$, Lijie Tan ${ }^{18}$, Danqing Li ${ }^{1}$, Qinghua \\ Zhou ${ }^{19}$, Xiangning $\mathrm{Fu}^{20}$, Zhongmin Jiang ${ }^{21}$, Haiquan Chen ${ }^{22,23}$, Wentao Fang ${ }^{23}$, Xun Zhang ${ }^{24}$, Yin $\mathrm{Li}^{25}$, Ti \\ Tong $^{26}$, Zhentao Yu ${ }^{27}$, Yongyu Liu ${ }^{28}$, Xiuyi Zhi ${ }^{29}$, Tiansheng Yan $^{30}$, Xingyi Zhang ${ }^{31}$, Andrea Imperatori ${ }^{32}$, \\ Mohsen Ibrahim ${ }^{33}$, Nuria M. Novoa ${ }^{34}$, Calvin Sze Hang $\mathrm{Ng}^{35}$, René Horsleben Petersen ${ }^{36}$, Jin-Shing \\ Chen $^{37}$, Yoshinosuke Fukuchi ${ }^{38}$, Alessandro Brunelli ${ }^{39}$, Mahmoud Ismail ${ }^{40,41}$, Javier Aragón Valverde ${ }^{42}$, \\ Carlos Rodriguez-Lucas ${ }^{43}$
}

${ }^{1}$ Department of Thoracic Surgery, Peking Union Medical College Hospital, Chinese Academy of Medical Sciences, Beijing 100730, China; ${ }^{2}$ Department of Thoracic Surgical Oncology, Cancer Institute \& Hospital, Chinese Academy of Medical Sciences and Peking Union Medical College; National Cancer Center, Beijing 100021, China; ${ }^{3}$ Department of Thoracic Surgery, China and Japan Friendship Hospital, Beijing 100096, China; ${ }^{4}$ Department of Cardiovascular and Thoracic Surgery, West China Hospital, Sichuan University, Chengdu 610041, China; ${ }^{5}$ Department of Thoracic Surgery, The First Affiliated Hospital of Guangzhou Medical University, Guangzhou 510230, China; ${ }^{6}$ Guangzhou Institute of Respiratory Disease \& China State Key Laboratory of Respiratory Disease \& National Clinical Research Center for Respiratory Disease, Guangzhou 510120, China; ${ }^{7}$ Department of Thoracic and Cardiovascular Surgery, The Third Affiliated Hospital of Kunming Medical University (Yunnan Tumor Hospital), Kunming 650118, China; ${ }^{8}$ Department of Thoracic Surgery, Harbin Medical University Cancer Hospital, Harbin 150086, China; ${ }^{9}$ Department of Thoracic Surgery, Zhejiang Cancer Hospital, Hangzhou 310022, China; ${ }^{10}$ Department of Thoracic Surgery, Institute of Surgery Research, Daping Hospital, Third Military Medical University, Chongqing 400042, China; ${ }^{11}$ Department of Thoracic Surgery, Fujian Medical University Union Hospital, Fuzhou 350001, China; ${ }^{12}$ Department of Thoracic Surgery, Tangdu Hospital, The Fourth Military Medical University, Xi'an 710038, China; ${ }^{13}$ Department of Thoracic Surgery, First Affiliated Hospital of Xinjiang Medical University, Urumqi, Xinjiang 830054, China; ${ }^{14}$ Department of Thoracic Surgery, Shanghai Pulmonary Hospital of Tongji University, Shanghai 200433, China; ${ }^{15}$ Department of Thoracic Surgery, Nanjing Medical University Affiliated Cancer Hospital, Jiangsu Key Laboratory of Molecular and Translational Cancer Research, Cancer Institute of Jiangsu Province, Nanjing 210009, China; ${ }^{16}$ Department of Thoracic Surgery, Sun Yat-Sen University Cancer Center, Guangzhou 510060, China; ${ }^{17}$ Department of Thoracic Surgery, Beijing Chao-Yang Hospital, Beijing 100020, China; ${ }^{18}$ Department of Thoracic Surgery, Shanghai Zhongshan Hospital of Fudan University, Shanghai 200032, China; ${ }^{19}$ Department of Lung Cancer Center, West China Hospital, Sichuan University, Chengdu 610041, China; ${ }^{20}$ Department of Thoracic Surgery, Tongji Hospital, Tongji Medical College, Huazhong University of Science and Technology, Wuhan 430030, China; ${ }^{21}$ Shandong Provincial Qianfoshan Hospital, Shandong University, Jinan 250014, China; ${ }^{22}$ Department of Thoracic Surgery, Fudan University Shanghai Cancer Center, Shanghai 200032, China; ${ }^{23}$ Department of Thoracic Surgery, Shanghai Chest Hospital, Shanghai Jiao Tong University, Shanghai 200030, China; ${ }^{24}$ Tianjin Chest Hospital, Tianjin 300051, China; ${ }^{25}$ Department of Thoracic Surgery, Henan Cancer Hospital, Zhengzhou 450008, China; ${ }^{26}$ Department of Thoracic Surgery, Second Hospital of Jilin University, Changchun 130041, China; ${ }^{27}$ Department of Esophageal Cancer, Tianjin Medical University Cancer Institute and Hospital, National Clinical Research Center for Cancer, Key Laboratory of Cancer Prevention and Therapy, Tianjin 300060, China; ${ }^{28}$ Department of Thoracic Surgery, Liaoning Cancer Hospital and Institute, Shenyang 110042, China; ${ }^{29}$ Department of Thoracic Surgery, Xuanwu Hospital of Capital Medical University, Beijing 100053 , China; ${ }^{30}$ Department of Thoracic Surgery, Peking University Third Hospital, Beijing 100083, China; ${ }^{31}$ Department of Thoracic Surgery, The Second Hospital of Jilin University, Changchun 130041, China; ${ }^{32}$ Center for Thoracic Surgery, Department of Medicine and Surgery, School of Medicine, University of Insubria, Varese, Italy; ${ }^{33}$ Division of Thoracic Surgery, Faculty of Medicine and Psychology, Sant'Andrea Hospital, University of Rome 'Sapienza', Rome, Italy; ${ }^{34}$ Thoracic Surgery Service, University Hospital of Salamanca, 37007 Salamanca, Spain; ${ }^{35}$ Department of Surgery, The Chinese University of Hong Kong, Prince of Wales Hospital, Shatin, NT, Hong Kong, China; ${ }^{36}$ Department of Cardiothoracic Surgery, University Hospital of Copenhagen, Rigshospitalet, Copenhagen, Denmark; ${ }^{37}$ Department of Surgery, National Taiwan University Hospital and National Taiwan University College of Medicine, 10002, Taipei; ${ }^{38}$ Department of Respiratory Medicine, Juntendo University, Tokyo, Japan; ${ }^{39}$ Department of Thoracic Surgery, St. James's University Hospital, Leeds, UK; ${ }^{40}$ Department of Thoracic Surgery, Klinikum Ernst von Bergmann, Potsdam, Germany; ${ }^{41}$ Academic Hospital of the Charité-Universitätsmedizin, Humboldt University Berlin, Berlin, Germany; ${ }^{42}$ Departament of Thoracic Surgery, 
Asturias University Central Hospital, Oviedo, Asturias, Spain; ${ }^{43}$ Department of Microbiology, Asturias University Central Hospital, Oviedo, Asturias, Spain

Correspondence to: Jie He, MD, PhD; Shugeng Gao, MD, PhD. Department of Thoracic Surgical Oncology, Cancer Institute \& Hospital, Chinese

Academy of Medical Sciences and Peking Union Medical College, National Cancer Center, Beijing 100021, China.

Email: hejie@cicams.ac.cn; gaoshugeng@vip.sina.com.

Submitted Feb 18, 2018. Accepted for publication Oct 29, 2018.

doi: $10.21037 /$ jtd.2018.10.108

View this article at: http://dx.doi.org/10.21037/jtd.2018.10.108

\section{Part 1: basic contents of the project}

\section{Project title}

Expert consensus on the use of antibacterial drugs in thoracic surgery.

\section{Aim and contents}

By establishing an expert consensus in this field, this study aims to develop a guide and uniform standard on the prophylactic and therapeutic use of antibiotics in clinical settings. This expert consensus aims to cover all the diseases that can be treated by antibacterial drugs in the thoracic surgery departments.

\section{Main issues}

This document is developed to address the nonstandard issues that may be encountered during the clinical use of antibacterial drugs.

\section{Part 2: background, status quo, and main research implications of the project}

Early in the 19th century, Pasteur and many other microbiologists had found that the occurrence and spread of some diseases were caused by microorganisms. However, in an era without any antibiotic drug, the infectious diseases raged on and claimed the lives of millions of people. For these tiny organisms that could only be seen under a microscope, people could do nothing. In 1928, Alexander Fleming's discovery of penicillin radically changed the fate of human civilization. It put an end to the history that there was no cure for infectious diseases and heralded the advent of antibiotics. It has been widely recognized as the third most important invention during the World War II, next only to the atomic bomb and radar. A variety of antibiotics have emerged in the following decades since, saving numerous lives. Today, infectious diseases are no longer as dreadful as they were in most countries and areas (1). However, along with the wide use and abuse of antibiotics, bacterial resistance has become a global concern. Early in the 19th century, Darwin indicated that creatures change to adapt to their environment to survive and thrive. His argument has been further confirmed by the changes in susceptibility of bacteria to antibiotics after decades of antimicrobial use. At present, bacterial resistance to antibiotics has gradually developed from the single-drug resistance to multiple-drug resistance; furthermore, these drug-resistant bacteria, once mainly seen in hospitals, have spread into communities via different manners (2). Once the speed of resistance development is faster than new drug research \& development, there will be no drug to control a specific bacterial infection. Take the evolution of bacterial resistance mediated by beta-lactamases as an example: the initially non-beta-lactamase-producing drug-susceptible bacteria have evolved into ultra-broad spectrum betalactamases- producing bacteria that are highly resistant to carbapenem (3). Pan-drug resistance has become a major threat to public health security.

The National Drug Resistance Report 2015 of China (4) revealed that 2,400,786 distinct bacterial strains [including 695,066 gram-positive strains (28.9\%) and 1,705,720 gram-negative strains (71.1\%)] were reported in 1,143 eligible hospitals (including 272 secondary hospitals and 871 tertiary hospitals). The top five gram-positive bacteria were Staphylococcus aureus (32.2\%), Staphylococcus epidermidis (12.8\%), Enterococcus faecalis (9.7\%), Streptococcus pneumoniae (9.3\%), and Enterococcus faecium (8.9\%). The top five gramnegative bacteria (GNB) were: Escherichia coli (29.9\%), Klebsiella pneumoniae (19.8\%), Pseudomonas aeruginosa (12.9\%), Acinetobacter baumannii (10.7\%), and Enterobacter cloacae (4.3\%). The top three specimen sources were sputum (41.4\%), urine (15.6\%), and blood (9.4\%). According to the 
monitoring data on bacterial drug resistance in China in the past four years, the detection rate of imipenem-resistant Acinetobacter baumannii was $58.0 \%$ in 2015, which showed a dramatic rise than that $(45.8 \%)$ in 2012 and was also higher than that $(54.5 \%)$ in 2014; the detection rate of imipenemresistant Klebsiella pneumoniae was $6.8 \%$ in 2015 , which was notably higher than that (4.8\%) in 2014; the detection rate of cefotaxime-resistant Escherichia coli showed no remarkable change between 2015 (60.3\%) and 2014 (60.0\%); the detection rates of other drug-resistant bacteria such as imipenem-resistant Escherichia coli, methicillin-resistant Staphylococcus aureus, vancomycin-resistant Enterococcus faecium, penicillin-resistant Streptococcus pneumoniae, and imipenem-resistant Pseudomonas aeruginosa were relatively stable.

For instance, among the 10,502 distinct bacterial strains detected in Peking Union Medical College Hospital (PUMCH) in 2015, the nine most common bacteria were Escherichia coli (15\%), Pseudomonas aeruginosa (9\%), Acinetobacter baumannii (9\%), Klebsiella pneumoniae (9\%), Staphylococcus aureus (8\%), Enterococcus faecalis (5\%), Enterococcus faecium (3\%), Candid albicans (3\%), and Enterobacter cloacae (2\%). The 3,324 bacterial strains detected in the respiratory tract included Pseudomonas aeruginosa (20\%), Acinetobacter baumannii (20\%), Klebsiella pneumoniae (13\%), Staphylococcus aureus (8\%), Stenotrophomonas maltophilia (4\%), Haemophilus influenzae (4\%), Aspergillus fumigatus (3\%), Escherichia coli (3\%), Enterobacter cloacae (3\%), and Streptococcus pneumoniae (2\%). Acinetobacter baumannii was extensively drugresistant and its susceptibility to tigecycline was only $55 \%$. The susceptibilities of Staphylococcus aureus to penicillin or erythromycin were only $10-30 \%$. The other isolated microorganisms included carbapenem-resistant Escherichia coli $(\mathrm{n}=32)$, carbapenem-resistant Klebsiella pneumonia $(\mathrm{n}=147)$, carbapenem-resistant Acinetobacter baumannii ( $\mathrm{n}=733)$, carbapenem-resistant Pseudomonas aeruginosa $(n=198)$, vancomycin-resistant enterococci $(n=33)$, methicillin-resistant Stapbylococcus aureus $(\mathrm{n}=211)$, ESBLpositive Escherichia coli ( $\mathrm{n}=849)$, ESBL-positive Klebsiella pneumoniae $(\mathrm{n}=180)$, and fungal infections $(\mathrm{n}=813)(5)$. In recent years, although the overall procurement volume of antibacterial drugs has not increased remarkably, the usage structure of these drugs has dramatically changed. The procurement volumes of 6 classes of antibacterial drugs (the first-, second-, and third-generation cephalosporins, nitroimidazoles, aminoglycosides, and glycopeptide) have shown a decline; in particular, the procurement volumes of the first- and second-generation cephalosporins have decreased by over $20 \%$. The antibacterial drugs with a procurement volume that has increased over $20 \%$ included amphenicols, antifungal agents for treating superficial infections, sulfonamides, tetracyclines, lincomycin, carbapenem, fourth-generation cephalosporins, macrolides, and penicillin/penicillinase inhibitors. The increase of the procurement volume was particularly obvious for the first three drugs listed (6). Consequentially, the pattern of bacterial resistance has changed significantly.

China is one of the countries with severe abuse of antibiotics. According to the World Health Organization, antibiotics are used in as high as $80 \%$ of hospitalized patients in China, which is far higher than the international level (30\%). Also, according to the Survey on Safe Drug Use among the General Public 2012 released by the China Food and Drug Administration (CFDA), nearly $40 \%$ of residents stored antibiotics in their home, $23.9 \%$ of residents chose to use antibiotics after a common cold, and only $40.1 \%$ of residents read the adverse reaction and cautions on package inserts and labels before using the drugs. Thus, drug abuse (in particular antibiotic abuse) remains an extremely severe issue in China. On August 11, 2010, an article in Lancet indicated that some bacteria with extraordinary antibiotic resistance have caused some relevant cases in In South Asia, Europe, and America and killed a few victims (7). On October 26, 2010, the Chinese Center for Disease Control and Prevention (China CDC) announced that three patients with New Delhi metallo-ß乃-lactamase (NDM)-1positive bacteria had been identified for the first time in China. On April 7, 2011, WHO marked the World Health Day on the theme "Antimicrobial resistance: no action today, no cure tomorrow". On April 18, 2011, the office of the Ministry of Health of China issued the Notification on Special Rectification Activities for the Clinical Application of Antibacterial Drugs, with an attempt to further strengthen the management of clinical application of antibacterial drugs in medical institutions, promote the rational use of antibacterial drugs, effectively control antimicrobial resistance, and thus ensure health care quality and patient safety. On May 6, 2011, the National Health and Family Planning Commission (formerly the Ministry of Health) kicked off these special rectification activities, which lasted three years (from 2011 to 2013); meanwhile, the commission released the Antibacterial Drugs Special Rectification Activity Protocol (for the years of 2011, 2012, and 2013) and the Administrative Measures for the Clinical Use of Antibacterial Drugs (come into effect after August 
1, 2012), which has achieved initial success. Monitoring in recent years has shown that the pathogen proportions and drug resistance of various infectious diseases in China have changed dramatically. Thus, the National Health and Family Planning Commission established a task force, headed by Dr. Nanshan Zhong, to revise the "Guiding Principles of Clinical Application of Antibacterial Drugs" [Methodist Medical (2004) No. 285] issued in 2004 after indepth research and extensive consultations. Based on these efforts, now the "Guiding Principles of Clinical Application of Antibacterial Drugs (2015 version)" are hereby promulgated to implement, so as to further standardize the clinical application of antibacterial drugs.

Currently the use of antibacterial drugs in the departments of thoracic surgery remains unstandardized, irrational, and controversial. The development of the expert consensus on the use of antibacterial drugs in this field was based on the following two considerations: first, the incidence of antimicrobial resistance has shown a rising trend worldwide; and second, the standardized clinical use of drugs will provide relatively uniform standards for the prophylactic and therapeutic use of antibiotics in the department of thoracic surgery, so as to instruct the thoracic surgeons to rationally use antibiotics; thus, it may help to address the nonstandard/irrational use of antimicrobial drugs in this discipline and thus reduce or delay the development of bacterial resistance.

\section{Part 3: main conclusions and recommendations}

According to the notification on the implementation of the "Guiding Principles of Clinical Application of Antibacterial Drugs" [Methodist Medical (2004) No. 285] issued by the Ministry of Health, the State Administration of Traditional Chinese Medicine, and the Health Department of PLA General Logistics Department, the basic principles of the prophylactic and therapeutic use of antimicrobial drugs are as follows:

\section{Basic principles of the prophylactic use of antimicrobial drugs}

\section{Antimicrobial prophylaxis in surgery \\ Aim}

To prevent post-operative incision infection, surgical site infection (SSI) following a clean-contaminated surgery or a contaminated surgery, and/or any possible systemic infection.

\section{Basic principles}

A decision on the antimicrobial prophylaxis should be made based on any contamination or possible contamination in the surgical field.

\section{(I) Clean surgeries}

The surgical field is in human sterile area, which has no local inflammation or injury and does not involve organs (e.g., respiratory tract, digestive tract, urinary and reproductive tract) that connect with the outside world. If the surgical field is not contaminated, antimicrobial prophylaxis is often not needed. However, it may be considered in the following conditions: (I) the surgery has wide range, long duration, and thus increased risk of contamination; (II) the surgery (e.g., skull surgery, cardiac surgery, and intraocular surgery) involves vital organs and may cause severe outcomes once an infection occurs; (III) implantation of foreign bodies such as prosthetic heart valve implantation, placement of permanent cardiac pacemaker, and prosthetic joint replacement; and (IV) surgeries performed in high-risk populations such as the elderly or immunocompromised persons.

\section{(II) Clean-contaminated surgeries}

Surgeries performed in the upper and lower respiratory tract, upper and lower digestive tract, or urogenital tract; or, surgeries (e.g., major surgeries via oropharyngeal cavity, transvaginal hysterectomy, and transrectal prostate surgery) performed via the above organs; or, surgeries for open fractures or trauma. Since these surgical sites are colonized by large numbers of a variety of bacteria, the surgical field may be contaminated during the surgery and thus cause infections. Therefore, antimicrobial prophylaxis is needed for these surgeries.

\section{(III) Contaminated surgeries}

During such operations, excessive overflow of body fluids from gastrointestinal tract or open wounds without debridement may cause the severe contamination of the surgical field. Thus, antimicrobial prophylaxis is needed for these surgeries.

\section{(IV) Selection and administration of drugs for} antimicrobial prophylaxis in surgery

The selection of antimicrobial drugs should be based on the aim of prophylaxis. Drugs against Staphylococcus aureus (S. aureus) should be selected to prevent postoperative SSI. For the purpose of preventing SSI or systemic infection, drugs against surgical field contamination or possible contaminating bacteria should be selected. For instance, antimicrobial drugs that are effective for E. coli and B. fragilis should be 
selected before a colon and rectal surgery. The selected antimicrobial drugs should have proven efficacy safe, easy to use, and relatively cheap.

\section{(V) Drug administration}

For patients undergoing a clean surgery, the drugs should be administered $0.5-2 \mathrm{~h}$ before surgery or upon the initiation of anesthesia, so that the drug concentrations in local tissues can be sufficiently high to kill bacteria contaminating the incisions during the surgery. If the operation lasts more than 3 hours or has massive blood loss $(>1,500 \mathrm{~mL})$, a second dose of the antimicrobial drug may be applied. The effective coverage time of the antimicrobial drug should include the whole surgical process and 4 hours after the end of surgery. The overall duration of the prophylaxis should be no more than 24 hours (may be extended to 48 hours in individual cases). For clean surgeries with relatively short surgical duration ( $<2$ hours), one dose of preoperative medication may be adequate. In patients receiving clean-contaminated surgeries, the duration of antimicrobial prophylaxis in surgery may also be 24 hours (may be extended to 48 hours if necessary). For contaminated surgeries, the duration of antimicrobial prophylaxis may be extended appropriately. In patients with preoperative infections, the duration of antimicrobial use may be based on the therapeutic use.

\section{Basic principles of the therapeutic use of antimicrobial drugs}

In patients with bacterial infections, antimicrobial drugs may be used only if indicated

Antimicrobials are only indicated if a diagnosis of bacterial infection is initially made based on symptoms, signs, and laboratory findings (blood and urine tests) or is confirmed after pathogen testing. Antimicrobials may also be indicated for infections caused by pathogenic microorganisms such as fungi, Mycobacterium tuberculosis, nontuberculous mycobacterium, mycoplasma, chlamydia, spirochetes, rickettsia, and some protozoa. Antimicrobials are not indicated in patients whose diagnoses cannot be established due to the lack of the evidences supporting the infections of a specific bacterium or the above pathogenic microorganisms or in patients with viral infections.

\section{After the early identification of pathogenic microorganisms, the antimicrobials should be selected based on the pathogen strains and the results of antimicrobial susceptibility testing}

In principle, the selection of antibacterial drugs should be based on pathogen strains and the results of antimicrobial susceptibility testing (for drug sensitivity or resistance). Therefore, in medical institutions with sufficient resources, specimens should be immediately collected from the inpatients for bacterial culture before the initiation of antimicrobial therapy to confirm the pathogen and antimicrobial susceptibility; for outpatients, antimicrobial susceptibility testing may be performed according to the disease conditions.

For critically ill patients, the most possible pathogen may be speculated based on symptoms/signs, onset setting, primary lesion, and underlying diseases before the information on pathogenic bacteria and drug susceptibility results becomes available. First attempting choice of antimicrobial drug should be directed by the local historical microbiological pathogenic records and drug resistance; then, empiric therapy using antibacterial drugs may be initiated based on local antimicrobial resistance. After the results of bacterial culture and drug susceptibility testing become available, the therapeutic regimen may be adjusted for patients who respond poorly to the initial treatment.

\section{Selecting antibacterial drugs according to the} antibacterial features of the drugs and their in vivo action mechanisms

Different antibacterial drugs have different pharmacodynamics (antimicrobial spectrum and antimicrobial activity) and pharmacokinetics (absorption, distribution, metabolism, and excretion of drugs) and thus have different clinical indications. Based on the above features of various antibacterial drugs, the clinicians shall correctly select the antibacterial drugs based on the clinical indications (see the "Indications and cautions of various antibacterial drugs").

\section{Antibacterial regimens should be formulated according to the disease conditions, pathogen types, and antibacterial features}

The antibacterial regimens (including antibacterial classes, dosage, administration frequency, administration route, treatment course, and combinations) should be established according to the pathogen, infection site, severity of infection, and physiopathological conditions of the patients. Establishment of the therapeutic regimens shall follow the following principles.

\section{Selection of antibacterial products}

The antibacterials shall be selected based on the results of bacterial culture and drug susceptibility testing. 


\section{Dosage}

Antibacterials shall be administered according to their therapeutic dose ranges. The dose of an antibacterial shall be relatively high (upper limit of the dosage range) for severe infections (e.g., septicaemia and infective endocarditis) and infections at the sites where an antibacterial can not easily reach (e.g., central nervous system infections); on contrast, a small dose may be applied (lower limit of the dosage range) for a simple urinary tract infection because the concentrations of most drugs in urine are far higher than blood drug concentrations.

\section{Route of administration}

(I) For patients with mild infection and feasible for oral administration, antibacterials that can be completely absorbed via oral routine shall be selected and no intramuscular or intravenous injection is needed. For patients with severe infection or systemic infection, the intravenous delivery should be applied during the initial treatment to ensure the drug efficacy; after the condition is improved and the oral administration becomes feasible, an early switch from intravenous to oral antibacterial is needed.

(II) Topical application of antibacterial drugs should be avoided: Antibacterial drugs locally applied on the skin and mucous membrane are seldom absorbed and can not reach the effective concentration in the infected area; rather, they may easily cause allergic reaction or lead to drug-resistant bacteria. Therefore, topical application of antibacterial drugs should be avoided for systemic infection or organ infection. The topical use of antibacterial drugs should only be allowed in rare cases; for instance, if the target treatment concentration cannot be reached in the infected site after systemic drug administration, topical use of an antibacterial may be feasible as an adjuvant therapy. An Acinetobacter infection with chest wall cellulitis from a thoracotomy infection: topical colistin can be applied to the wounds. Infections in the outer layers of the skin or on the mucosal surfaces (especially oral and vaginal mucosal surfaces) can be treated by the local or topical use of antimicrobial drugs; however, topical use of drugs mainly for systemic disease should be avoided. The topically used drugs should be antimicrobial drugs that are less irritating, not easy to absorb, not easy to develop bacterial resistance, and not easy to cause allergic reaction. Penicillins, cephalosporins, and other antibiotics that can easily cause allergic reaction should not be topically applied. Ototoxic drugs (e.g., aminoglycosides) should not be prescribed as antibiotic eardrops.

\section{Drug administration frequency}

Drugs should be administered based on both pharmacokinetics and pharmacodynamics with an attempt to maximize the drug efficacy in human body and kill pathogenic bacteria in the infection site. Penicillins, cephalosporins, and other $\beta$-lactams, erythromycin, and clindamycin having a short elimination half-life should be administered multiple times per day. Fluoroquinolones and aminoglycosides can be administered once daily (sometimes, this cannot be applicable for patients with severe infections).

\section{Treatment course}

The treatment courses of antibacterial drugs vary due to different infections. Typically the treatment should continue 72-96 hours after the body temperature becomes normal and the symptoms disappear. Special conditions should be properly managed. However, pleural empyema, sepsis, infective endocarditis, purulent meningitis, typhoid, brucellosis, osteomyelitis, hemolytic streptococcal pharyngitis and tonsillitis, deep mycoses, tuberculosis, and other diseases require a longer treatment course for achieving a thorough cure and preventing recurrence.

\section{Combination of antibacterial drugs requires specific indications}

No drug combination is required if a single drug can effectively treat an infection. Drug combination is only needed in the following conditions:

(I) Severe infections with unknown causes, including severe infections in patients with immunodeficiency;

(II) Mixed aerobic and anaerobic infections (two or more pathogens) that cannot be controlled by a single antibacterial drug;

(III) Severe infections (e.g., infective endocarditis or sepsis) that cannot be effectively controlled by a single antibacterial drug;

(IV) Infections (e.g., TB and deep mycoses) requiring long-term treatment but the pathogen can easily develop antibacterial resistance to certain drugs;

(V) Due to the presence of synergistic antibacterial activities, antibacterial drugs with higher toxicities should be used at lower doses when the drugs are used in combination. For instance, when amphotericin $\mathrm{B}$ and flucytosine are used in 
combination to treat cryptococcal meningitis, the dose of amphotericin B may be lowered to reduce its toxic reactions. When drugs are given in combination, drugs with synergistic or additive antibacterial effects should be selected. For example, penicillins, cephalosporins, other $\beta$-lactam antibiotics may be used in combination with aminoglycosides, whereas amphotericin B may be combined with flucytosine. Two-drug combinations are most commonly applied; combinations based on three or more drugs are only required in less common conditions (e.g., treatment of TB). It is also notable that adverse drug reactions will increase after antibacterial drugs are used in combination.

\section{Use of antibacterial drugs in thoracic surgery}

SSI is a common nosocomial infection. The presence of SSI increases the in-hospital mortality and prolongs hospital stay. Preoperative antibiotic prophylaxis has been well recognized as a useful strategy for lowering the incidence of SSI. However, the optimal timing of preoperative antibiotic prophylaxis has not been defined. The most widely accepted view is: the time for antibiotic prophylaxis is before the skin is cut open, i.e. two hours before surgery; in addition, its effectiveness in preventing SSI is better when the timing is closer to the skin incision. Thus, currently the antibiotic prophylaxis is typically performed $30 \mathrm{~min}$ before surgery.

Antibiotic prophylaxis is often not required for the clean surgery; when it is applied, the indications, drug selection, and treatment initiation and duration must be strictly controlled. The drug administration must follow the guiding principles for the clinical application of antibacterial drugs. The first dose should be $0.5-2$ hours before surgery or at anesthesia induction; if the duration of surgery exceeds 3 hours or if the intraoperative blood loss is larger than $1,500 \mathrm{~mL}$, a second dose can be applied during the surgery. The overall duration of antibiotic prophylaxis usually lasts no more than 24 hours but may be extended to 48 hours in individual cases (8). Typically the second-generation cephalosporins are selected for antibiotic prophylaxis. Selection of the antibacterial drugs and the timing of drug usage should be decided based on the disease conditions. For suspected infections, corresponding microbiological examinations need to be performed; if required, drug susceptibility testing may also be conducted $(8,9)$.

\section{Pharmaceutical notes (8)}

(I) Antibiotic prophylaxis is contraindicated in patients with a history of an allergic reaction to cephalosporins of any kind and/or a history of anaphylactic shock following penicillin use.

(II) Before the medication, any previous history of allergy to cephalosporins, penicillins, or other drugs should be inquired about in details. For patients with a history of allergy to penicillin, other $\beta$-lactam antibiotics, and other drugs, this kind of drugs should be used with caution even when there are clear indications. If allergic reaction occurs, the medication must be stopped immediately. If anaphylactic shock occurs, rescue treatment should be provided immediately on the spot, along with adrenaline administration and other treatments.

(III) This class of drugs is mainly excreted through kidney. The dosage must be appropriately adjusted according to kidney function in patients with moderate or severe renal insufficiency.

\section{Intraoperative antibiotic prophylaxis}

(I) Antibiotic prophylaxis in principle is not applied in type I incision operations (surgeries that do not involve esophagus, cardia, trachea, or bronchi) such as substernal thyroid surgery, video-assisted thoracoscopic (VATS) mediastinal tumor resection, and mediastinoscopic biopsy; however, if planned surgery duration is longer than 3 hours or if the intraoperative blood loss is more than $1,500 \mathrm{~mL}$, a second antibiotic prophylaxis may be added.

(II) Preoperative antibiotic prophylaxis may be applied for median sternotomy for mediastinal tumor resection (surgeries that do not involve esophagus, cardia, trachea, or bronchi), chest wall surgery, and VATS pulmonary wedge resection.

(III) For the VATS lobectomy, the first dose has to be administered 0.5-1 hours before surgery (before the skin incision, at induction of anesthesia) or when anesthesia begins; if the duration of surgery exceeds 3 hours or if the intraoperative blood loss is larger than $1,500 \mathrm{~mL}$, a second dose can be applied during the surgery. The overall duration of antibiotic prophylaxis usually lasts no more than 24 hours but may be extended to 48 hours in individual cases. A special consideration has to be taken into account in Uniportal VATS because the drain remains in place across the wound. Experts 
recommend to maintain antibiotic prophylaxis until the drain is removed. In patients with early postoperative complications such as bronchopleural fistula, significant pulmonary air leak, atelectasis, and/or chylothorax, the duration of antibiotic prophylaxis may be prolonged where appropriate. Meanwhile, the reason for such prolonging should be documented in medical records and the pleural effusion specimen was collected for etiological examination. If there are infection-related symptoms/ signs such as elevated body temperature/blood cell amounts, empiric antibacterial treatment shall be applied, along with blood culture, sputum culture, and drainage culture. The drug use may be adjusted after the drug susceptibility test results become available.

(IV) For patients undergoing an open pulmonary wedge resection or lobectomy, preoperative antibiotic prophylaxis should be performed once, and the postoperative antibiotic prophylaxis should be administered for 48 hours (8). In patients with postoperative complications such as bronchopleural fistula, long-term pulmonary air leak, atelectasis, and/or chylothorax, the duration of antibiotic prophylaxis may be prolonged where appropriate. Meanwhile, the reason for such prolonging should be noted in medical records and the pleural effusion specimen collected for etiological examinations.

(V) For patients undergoing clean-contaminated surgery (surgeries that involve esophagus, cardia, trachea, or bronchi), preoperative antibiotic prophylaxis should be performed once, and the postoperative antibiotic prophylaxis should be administered for 72 hours. For patients undergoing surgery for removing esophageal leiomyoma or for treating benign esophageal diseases such as achalasia and hiatal hernia, the duration of postoperative antibiotic prophylaxis should be no more than 24 hours if the digestive tract mucosa is not damaged during the surgery; however, if there is intraoperative digestive tract mucosa damage, the duration of postoperative antibiotic prophylaxis should be prolonged accordingly.

(VI) The duration of postoperative antibiotic prophylaxis may also be prolonged (in principle no more than 72 hours) in patients with susceptible underlying diseases such as bronchiectasis, COPD, and diabetes; the reason for prolonging antibiotic use should be noted in the medical records.

\section{Drugs selected for antibiotic prophylaxis}

(I) For pulmonary and mediastinal surgeries

In patients without underlying lung diseases, the second-generation cephalosporins (e.g., cefuroxime $1.5 \mathrm{~g} \mathrm{q} 8 \mathrm{~h}$ iv drip/iv/im), cephamycins (e.g., cefmetazole, 1-2 g q12h iv drip/iv) may be selected.

(i) For patient with underlying lung diseases such as bronchiectasis, the third-generation cephalosporins (e.g., ceftriaxone 1-2 g qd im/iv or $0.5-1$ g q12h $\mathrm{im} / \mathrm{iv}$ ), quinolones (e.g., levofloxacin 250-500 mg iv drip qd and moxifloxacin $0.4 \mathrm{~g}$ iv drip qd) may be selected; or, the drugs may be selected based on the results of bacterial culture (sputum culture and/ or bronchoscopic brushing) and drug susceptibility tests.

(ii) For penicillin- or cephalosporin-allergic patients, clindamycin and fluoroquinolones (e.g., levofloxacin $250-500 \mathrm{mg}$ iv drip qd or moxifloxacin $0.4 \mathrm{~g}$ iv drip qd) may be selected.

(iii) For patients with a history of confirmed or suspected old tuberculosis, fluoroquinolones (e.g., levofloxacin $250-500 \mathrm{mg}$ iv drip qd or moxifloxacin $0.4 \mathrm{~g}$ iv drip qd) may be selected.

\section{(II) For clean-contaminated surgery for esophagus and} cardia

(i) The second-generation cephalosporins (e.g., cefuroxime $1.5 \mathrm{~g}$ q8h iv drip/iv/im), cephamycins (e.g., cefmetazole, 1-2 g q12h iv drip/iv), or the third-generation cephalosporins (e.g., ceftriaxone $1-2 \mathrm{~g}$ qd im/iv or $0.5-1 \mathrm{~g} \mathrm{q} 12 \mathrm{~h} \mathrm{im} / \mathrm{iv}$ ) in combination with anti-anaerobic bacteria agents (e.g., metronidazole: first dose: $15 \mathrm{mg} / \mathrm{kg}$; maintenance dose: $7.5 \mathrm{mg} / \mathrm{kg} \mathrm{q} 8 \mathrm{~h}$ iv drip).

(ii) For penicillin- or cephalosporin-allergic patients, monotherapy with a broad-spectrum antibiotic can be selected: aztreonam, quinolones (e.g., levofloxacin 250-500 $\mathrm{mg}$ iv drip qd), and carbapenems (e.g., ertapenem $1 \mathrm{~g}$ qd iv drip/im).

\section{Therapeutic use of antibiotics}

(I) Empiric use of antibiotics (pathogens should be identified via multiple tests in a timely manner, along with drug susceptibility test):

(II) For infections unrelated to the digestive tract (including infections in the lungs, pleura, mediastinum, and chest wall): The secondgeneration cephalosporins (e.g., cefuroxime 
0.75-1.5 g q8h iv drip/iv), cephamycins (e.g., cefmetazole, $1-2 \mathrm{~g}$ q12h iv drip/iv), quinolones (e.g., levofloxacin 250-500 $\mathrm{mg}$ iv drip qd), and moxifloxacin hydrochloride tablets $0.4 \mathrm{~g}$ iv drip qd).

(III) In patients with signs/symptoms of infection (e.g., fever, purulent sputum, and elevated white blood cells and neutrophils), broad-spectrum antibacterial drugs (e.g., the third-generation cephalosporins or fluoroquinolones) should be selected accordingly.

(IV) For infections related with digestive tract: The third-generation cephalosporins (e.g., ceftriaxone 1-2 g qd im/iv or $0.5-1 \mathrm{~g} \mathrm{q} 12 \mathrm{~h}$ im/iv, ceftazidime 4-6 g q12h iv/iv drip), aztreonam (1-2 g q8h/q6h iv/iv drip/im), carbapenems (e.g., ertapenem $1 \mathrm{~g}$ qd iv drip/im), and combinations with beta-lactamase inhibitors (augmentin for injection $1.2 \mathrm{~g} \mathrm{q} 8 \mathrm{~h}$ iv/iv drip; tazocin $4.5 \mathrm{~g} \mathrm{q} 8 \mathrm{~h}$ iv/iv drip).

(V) For patients with a special infection, specific drug treatment should be applied according to its features. For instance, patients with accompanying TB should receive anti-TB treatment first.

After the results of pathogen tests and drug susceptibility tests become available, more sensitive antibiotics should be used instead; also, the duration of antibacterial drug use should be prolonged and the drug types should be switched if necessary.

\section{Pharmaceutical notes}

(I) Any history of drug allergies should be carefully inquired about before medication administration.

(II) Before drug use, any possible effect of the drug on liver and kidney functions should be taken into consideration and the dosage should be timely adjusted. Pay attention to the nephrotoxicity and ototoxicity of aminoglycosides (8). When quinolones are used in patients with renal insufficiency, the dose should be lowered or the duration of drug administration should be prolonged based on creatinine clearance rate (8).

(III) Drug-drug interactions should be taken into consideration. For instance, macrolides can interact with methylprednisolone, theophylline, carbamazepine, and warfarin.

(IV) Pay attention to the doses, timing, and administration routes of drugs.

(V) Pay attention to the different applications of drugs in children, pregnant women, and the elderly.

\section{Cautions $(9,10)$}

(I) For antibacterial drugs with a primary drug- resistance rate of higher than $30 \%$, remind the medical staff to pay attention to such drugs;

(II) For antibacterial drugs with a primary drugresistance rate of higher than $40 \%$, use such drugs cautiously;

(III) For antibacterial drugs with a primary drugresistance rate of higher than $50 \%$, use such drugs according to the results of drug susceptibility tests;

(IV) For antibacterial drugs with a primary drugresistance rate of higher than $70 \%$, suspend the clinical application of such a drug for the targeted bacterium; whether the clinical use of such a drug should be re-initiated should be based on the results of drug susceptibility tests.

\section{Part 4: main issues-ongoing controversies and relevant research}

\section{Multidrug-resistant (MDR) bacteria}

\section{Emergence of MDR bacteria}

Before 1940s, human beings are long waiting for a drug that can effectively treat bacterial infections with neglectable side effects. The discovery of penicillin greatly enhanced the patient's capability to fight against bacterial infections. Since then, a large amount of antibiotics have been developed; over a long period of time, these antibiotics have greatly increased the cure rates of infections. However, antibiotic abuse has become an increasingly severe issue along with the wide use of antibiotics. About $50 \%$ of antibiotics are abused each year around the world; in China this figure even approaches $80 \%$. Due to the abuse of drugs, bacteria adapt quickly to antibiotics in the environment, resulting in the emergence of various drug-resistant bacteria. Currently, among ICU patients with severe infections, the common pathogens of ventilator-associated pneumonia (VAP) include extended-spectrum beta-lactamases (ESBLs), Klebsiella pneumoniae carbapenemase (KPC), Stenotrophomonas maltophilia Pseudomonas; and methicillin-resistant Staphylococcus aureus (MRSA). The emergency of MDR bacteria not only urges the research and development of new antibiotics, but also provides a warning message for the irrational use of antibiotics.

\section{Status quo of management of MDR bacteria}

If a MDR bacterial infection is highly suspected, antibiotic use should be initiated immediately because any delay or improper treatment will increase the morbidity or mortality. However, only one third of VAP patients displayed the 
clinical manifestations of sepsis; in patients without any sign of severe sepsis or septic shock and if gram staining showed no microbes, the antimicrobial therapy may be postponed till the culture reports become available.

According to current guidelines, antibiotics for the empiric treatment of GNB include: the third-/fourthgeneration of cephalosporins, piperacillin/tazobactam, and carbapenem combined with fluoroquinolones or aminoglycosides. However, currently a high proportion of GNB have become resistant to commonly used antibiotics. As a result, there are very few antibiotics that can be used for treating MDR bacterial infections.

\section{New research findings and possible problems}

\section{Antibiotics for MDR-GNB \\ Carbapenems}

Carbapenems have been used for many years. The commonly used carbapenems include imipenem, meropenem, and doripenem. They have similar antimicrobial spectrum, although doripenem is most effective for Pseudomonas aeruginosa (11). Currently the possible improvement protocols include: (I) to extend the infusion time of carbapenems. Research has shown that the extended infusion protocol can achieve better pharmacokinetics and improve the clinical outcome of VAP $(12,13)$. (II) Use of dual carbapenem therapy. Literature has demonstrated that the dual carbapenem therapies (ertapenem plus meropenem or doripenem) have been explored in animal experiments. The KPC enzymes prefer to bind ertapenem, which hinders the degrading of doripenem or meropenem and thus facilitates the use of such carbapenem. Currently this strategy has been applied in VAP patients with KPC infection resistant to all drugs with satisfactory outcomes (14).

\section{Colistin}

Currently, colistin is the most effective in vitro treatment for MDR-GNB (including Acinetobacter baumannii, Pseudomonas aeruginosa, extended spectrum beta-lactamase (ESBL)-producing Enterobacteriaceae, and carbapenem enzyme-producing Klebsiella) (15); however, some GNB including Proteus, Providencia, Morganella, and Serratia marcescens may be resistant to colistin.

Although empiric treatment using the combination of colistin and rifampicin has been applied in different GNB, recent randomized controlled trials have shown that in patients with VAP due to MDR Acinetobacter baumannii or carbapenem-resistant Acinetobacter baumannii, such combination showed no significant difference with colistin monotherapy (16). However, in vitro research has shown that colistin plus glycopeptide is effective in treating carbapenem-resistant Acinetobacter baumannii (17).

In a retrospective study on Acinetobacter baumannii, we did not find that the combination of colistin and glycopeptide was superior to colistin monotherapy; rather, it increased the risk of renal failure (18). On the contrary, a multi-center study revealed that, for infections caused by different GNB, the duration of the combination therapy (colistin plus glycopeptide) of less than 5 days was a protective factor for the 30-day mortality (19).

\section{Tigecycline}

As a broad-spectrum antibiotic for anaerobic and aerobic gram-positive and -negative pathogens (excluding Pseudomonas aeruginosa and Proteus), tigecycline is effective in treating Acinetobacter baumannii (including some colistin-resistant strains) (20).

Tigecycline has been approved for the treatment of abdominal infections and skin/soft tissue infections (50 mg/12 h, with a loading dose of $100 \mathrm{mg}$ ); however, this dosage is not high enough to reach the concentration required for lung infection). A recent randomized phase II trial has evaluated the clinical efficacies of two highdose protocols (tigecycline $v s$. imipenem/cilastatin) in treating nosocomial pneumonia. Although the findings were not conclusive due to small sample size, the dose of $100 \mathrm{mg} \mathrm{q} 12$ with a loading dose of $200 \mathrm{mg}$ achieved the maximal clinical cure (21). Thus, in patients with suspected highly resistant GNB infections, high-dose antibiotics may be applied to avoid insufficient dose in lung tissue; generally, according to our experiences, a combination (with carbapenem or colistin) may be the option of choice.

\section{Fosfomycin}

An old antibiotic, previously used for the treatment of gram-positive and -negative bacterial infections, with small toxicities; however, drug resistances can easily development following monotherapy. A combination with other drugs enables fosfomycin to be used for more infections, including MDR VAP.

Due to the lack of effective alternate treatment, fosfomycin has been applied in the treatment of extensively drug-resistant (XDR) GNB, especially Klebsiella pneumoniae and Pseudomonas aeruginosa. A series of studies have reported that high-dose fosfomycin $(24 \mathrm{~g} / \mathrm{d})$ could achieve satisfactory response in VAP patients when combined with other antibiotics. 
Antibiotics for gram-positive cocci

\section{Teicoplanin}

Teicoplanin may be an acceptable alternate drug for pneumonia caused by MRSA. However, high-dose teicoplanin need to be carefully administered $(12 \mathrm{mg} / \mathrm{kg}$ q12h for the first 48 hours, followed by $12 \mathrm{mg} / \mathrm{kg}$ on a daily basis) to make the antibiotic level in lung tissue reach a stable level (22). Teicoplanin is not recommended for VAP because the dosage cannot be determined, the serum drug level cannot be monitored, and there are several alternate protocols such as vancomycin or linezolid.

\section{Linezolid}

Linezolid has better pharmacokinetic properties than vancomycin. Two randomized double-blind study came to the same conclusion: in one arm, the clinical cure and survival rates of patients with VAP caused by MRSA were significantly better in linezolid group than in vancomycin group (23). A recent double-blind controlled clinical trial revealed that, for pneumonia caused by MRSA, although vancomycin could optimize the dose and reduce renal toxicity, linezolid had higher clinical and microbiological response rates (the latter had no statistical significance) (24). For MRSA, however, the number of cases clinically cured by tigecycline is smaller than the expected number based on clinical trials (25). Thus, tigecycline is not recommended for pneumonia caused by MRSA, and a specific antibiotic is needed to fight against this gram-positive bacterium.

\section{Ceftaroline}

Ceftaroline is a broad-spectrum cephalosporin against gram-positive bacteria (including MRSA), whereas it is also useful for some GNB including the commonly seen pathogenic bacteria in the respiratory tract and enterobacteriaceae. However, currently ceftaroline is only approved for the treatment of acute bacterial skin infections and community-acquired pneumonia. This new cephalosporin has a promising role in the treatment of VAP; however, currently no clinical data are available. It should be combined with another antibiotic to cover GNB such as Pseudomonas aeruginosa or ESBL-producing enterobacteriaceae.

\section{Aerosolized antibiotics}

Aerosolized antibiotics can achieve higher drug concentrations in lung tissue and reduce systemic injuries. Although many antibiotics can be aerosolized, the most commonly used aerosolized antibiotics are aminoglycosides and colistin. The aerosolized colistin can increase the cure rate of pneumonia due to MDR-GNB and may be effective and safe (26). In a retrospective study, the aerosolized colistin was independently correlated with clinical cure (OR, 2.53, 95\% CI: 1.11-5.76), although the mortality showed no significant change. However, some other research concluded that the aerosolized and intravenous colistin had no additional benefit for GNB-MDR VAP. For aminoglycosides, several studies have demonstrated the clinical values of tobramycin and kanamycin A sulfate (amikacin).

\section{Duration of antibiotic use}

No consensus has been reached for the duration of antibiotic treatment for MDR-VAP. In a randomized controlled trial, adequate empiric antibiotic therapy was applied in VAP patients and it was found that the casefatality rate was not significantly different between the short-course (8 days) and the long-course (15 days) groups. However, the recurrence rate was significantly higher in patients with VAP caused by non-fermentative gramnegative bacilli (such as Pseudomonas aeruginosa) (27). Meta-analysis of four recent randomized controlled trials also concluded that short-course antibiotic regimens are sufficient for VAP; however, the duration for MDR-VAP remained indefinite (28). A prospective study showed that procalcitonin-based strategies (stop using antibiotics when PCT $<0.5 \mathrm{ng} / \mathrm{mL}$ or reduced by $\geq 80 \%$ ) did not have negative impact, although there was no definite assessment on the MDR-VAP subgroup (29).

\section{Drugs for the treatment of MDR-tuberculosis (MDR-TB) and possible problems}

MDR-TB refers to the tuberculosis caused by Mycobacterium tuberculosis isolates that are resistant to isoniazid and rifampicin and may also become resistant to other chemotherapy drugs. MDR-TB includes both primary resistance (previously have not received anti-TB treatment but have been infected with drug-resistant Mycobacterium tuberculosis strains) and secondary resistance (e.g., the patients have been infected with a drug-sensitive strain but such a strain develops drug resistance during or after the treatment). However, few controlled trials have explored the MDR-TB treatment. The first-line drugs for drug-sensitive TB strains include isoniazid, rifampicin, ethambutol, and pyrazinamide; the second-line drugs for drug-resistant $\mathrm{TB}$ are mainly fluoroquinolones and aminoglycosides for 
injection; other less effective second-line drugs include ethionamide, cycloserine, and aminosalicylic acid. Some other drugs with less clinical data include clofazimine, amoxicillin/clavulanic acid, linezolid, carbapenem, thioacetazone, and clarithromycin. The main side effects are liver and kidney toxicities. Now there are also some new drugs, as represented by bedaquiline. Among 440 MDR-TB patients enrolled in two phase II clinical trials, compared with patients who had only received background regimens, patients who had received bedaquiline in combination with optimal background regimens had quicker sputum negative conversion and high negative conversion rate $(77.6 \% \mathrm{vs}$. $57.6 \%)(30,31)$.

However, FDA approved bedaquiline with a blackbox warning, indicating that this drug may cause QT prolongation and thus result in arrhythmia or death. According to the data submitted to FDA, compared with patients who had only received background therapy, patients who had received bedaquiline combination were more likely to die (11.4\% vs. $2.5 \%)$; the most common cause of death was TB.

In October 2013, the US CDC issued interim guidance on the use of bedaquiline, recommending that clinical expert advice and directly observed therapy (DOT) should be used as parts of the comprehensive case management strategy for TB. The guidance also extended the use of bedaquiline in patient populations that had not been included in clinical trials and thus had not been approved by FDA; these patient populations include children, pregnant women, extrapulmonary MDR-TB patients, and patients with HIV infection or other co-morbidities. According to CDC, although the safety and effectiveness of bedaquiline in these populations have not been demonstrated, bedaquiline still can be used based on the specific conditions of the patients when there is no other effective treatment regimen.

In 2014, 160 smear-positive adult MDR-TB patients were randomly allocated to receive the second-line background treatment plus bedaquiline (400 $\mathrm{mg}$, qd, for 2 consecutive weeks; followed by $200 \mathrm{mg}$, three times per week, for 22 consecutive weeks) or placebo (32). Most patients were HIV-negative, and $43 \%$ of the screened individuals were excluded due to some other factors (e.g., alcoholism, pregnancy, and prolonged QT interval). Compared with the placebo group, the bedaquiline group had significantly shorter time required for sputum negative conversion (83 vs. 125 days); also, bedaquiline group had higher negative conversion rates in the 24 th week (79\% vs. $58 \%)$ and in the 120 th week (62\% vs. $44 \%)$; however, the case-fatality rate was also higher in the bedaquiline group, with unknown causes. These findings suggest that bedaquiline may have a role in treating specific patients with MDR-TB; however, more clinical trials are needed to assess its death risks and efficacy in HIV carriers.

Other new drugs such as delamanid, which can inhibit the synthesis of Mycobacterium tuberculosis, and PA-824, which can sterilize the pathogen, have been developed. However, the optimal combinations of these drugs in clinical setting require further investigations.

\section{Use of antifungal drugs}

Fungal infection mainly occurs in immunocompromised hosts, whereas neutropenia and use of glucocorticoids are the most common predisposing factors. However, fungi may also develop drug resistance. Along with the increase of antifungal drugs, drug combinations and their roles in fungal prevention and early treatment have increasingly been recognized.

\section{Combination therapy}

Up to now the roles of combined antifungal therapies have only been explored in patients who failed to respond to initial treatment. However, the values of combined antifungal therapies for initial treatment or salvage treatment have not been well documented. Therefore, the enhanced efficacies of drug combinations must be balanced with the increased toxicities and their potential antagonistic effects (33).

\section{Prevention and early treatment}

Up to now many favorable results have been obtained from the randomized trials on the role of antifungal drugs in preventing invasive aspergillosis; however, the preventive measures themselves have potential pitfalls such as the potential toxicities and drug interactions, drug pricing, and the possible microbial drug resistance. In patients with gastrointestinal mucositis and/or graft versus host disease (GVHD), absorption of oral drugs is often difficult (34). It is still not clear which patient populations might benefit from the preventive treatment, which partially depends on the patient characteristics and the epidemiology of invasive fungal infections in the body. Preemptive antifungal therapy, as an early treatment strategy, has been proposed to be an alternative treatment for empiric therapy. For a preemptive antifungal therapy, the antifungal therapy is initiated based on the results of a series of screening for aspergillosis. Ongoing randomized trials are exploring the 
value of preemptive antifungal therapy. Although a study has suggested that the preemptive antifungal therapy only had limited benefits, no definite conclusion has been drawn due to limitations in study design. The safety and effectiveness of preemptive antifungal therapy in patients with decreased neutrophil is still unclear; therefore, preemptive antifungal therapy is not recommended.

\section{Medication-related problems in patients with abnormal liver function}

The use of antibiotics may be problematic in patients with abnormal liver functions, in whom the following issues must be addressed firstly.

\section{Role of infection in the abnormal liver function}

If liver function damage is caused by an infection, the abnormal liver function is less important in the selection of anti-infective drugs; while the selected highly specific antibiotic may damage the liver, it may still be beneficial to alleviate the abnormal liver function.

\section{Influence of abnormal liver function on the pharmacokinetics of antibiotics}

Mechanisms via which the liver disease affects the pharmacokinetics of antibiotics: (I) reduced hepatic drug clearance; (II) decreased protein binding; (III) increased drug distribution volume; (IV) altered absorption of oral medications; and $(\mathrm{V})$ inductive effect of some drugs on hepatic drug-metabolizing enzymes.

These mainly include the following conditions:

(I) For non-hepatotoxic drugs that are mainly secreted through the kidneys, normal doses can be used in patients with liver dysfunction. These antibiotics include penicillin, aminoglycosides (gentamicin, tobramycin, and amikacin), cefotaxime, vancomycin, and colistin.

(II) For non-hepatotoxic drugs that are mainly cleared via the liver, the clearance of the drug may slow down or decrease in patients with liver dysfunction. Thus, these drugs must be used with caution or at a lower dose level. These antibiotics include lincomycin, clindamycin, and erythromycin (excluding esterified erythromycin); the blood drug concentration should be monitored if condition allows.

(III) For drugs cleared via liver and kidneys, the drug clearance can decrease in patients with liver dysfunction, along with increased blood drug concentration. In patients with accompanying kidney dysfunction, the increased blood drug concentration can be more obvious, which can easily cause liver damage. Such drugs may be used at reduced doses. These drugs include ureidopenicillins (including azlocillin and mezlocillin) and cephalosporins (e.g., cefoperazone, ceftriaxone, and cefotaxime).

(IV) For hepatotoxic drugs that are mainly cleared via the liver, the decreased drug clearance in patients with liver dysfunction can lead to toxic reactions. These drugs include esterified erythromycin, rifampicin, isoniazid, tetracyclines, sulfonamides, chloramphenicol, amphotericin B, ketoconazole, and miconazole.

\section{Avoid using antibacterial drugs with high risk of liver injury \\ $\beta$-lactam antibiotics}

\section{(I) Penicillin}

Amoxicillin monotherapy can sometimes be mildly hepatotoxic, whereas oxacillin, carbenicillin, and ticarcillin can be associated with transient elevation of alanine transaminase (ALT). The incidence of flucloxacillinrelated liver damage is about $1.8 / 100,000$ or $2.6 / 100,000$, and the incidence of flucloxacillin-related jaundice is about 3.6/100,000. Research has shown that the risk of flucloxacillin-related jaundice increased in elderly patients and patients who have used the drug for more than two weeks.

\section{(II) Amoxicillin/clavulanic acid}

The combination of amoxicillin and clavulanic acid can significantly increase the risk of liver toxicity, and $13-23 \%$ of drug-mediated liver injury is caused by amoxicillin/ clavulanic acid. Early diagnosis can be difficult because the symptoms often appear later.

\section{(III) Cephalosporins}

Rare.

\section{Macrolides}

\section{(I) Erythromycin}

Erythromycin-induced liver injury was reported more than 40 years ago and has been reported in its various derivatives. Recent studies have shown that the incidences of the liver toxicities of erythromycin are similar to those of penicillin $\mathrm{V}$.

\section{(II) Clarithromycin}

The risk of the liver toxicity of clarithromycin is similar to 
that of erythromycin.

\section{(III) Azithromycin}

Rare. Rare cases with intrahepatic cholestasis have also been reported.

\section{Quinolones}

\section{(I) Ciprofloxacin}

Few studies have explored the hepatotoxicity of ciprofloxacin. Currently it is believed that the incidence of its hepatotoxicity is low.

\section{(II) Levofloxacin}

Levofloxacin also has a low incidence of hepatotoxicity. Clinical research has shown that less than $1 \%$ of patients developed abnormal liver function after the use of levofloxacin and the incidence of severe hepatotoxicity was below $1 / 5,000,000$ cases.

\section{(III) Moxifloxacin}

About $1-5 \%$ of patients may develop liver dysfunction following the use of moxifloxacin; however, research has shown that moxifloxacin almost has no risk of liver damage.

\section{Sulfonamides}

The hepatotoxicity of sulfonamides has already been described. According to a study performed in British populations, about 1/1000 of patients developed liver toxicity after the use of sulfonamides, and thus sulfonamides are among the most hepatotoxic drugs.

\section{Tetracyclines}

The hepatotoxicity of tetracyclines was reported 50 years ago, which might be related with high-dose tetracycline use. Unlike other antibiotics, the hepatotoxicity of tetracyclines can be reproduced and predicted in animal models. Oral tetracyclines in normal doses seldom cause hepatotoxicity (about 1/1,800,000 cases).

\section{Linezolid}

Extended duration of treatment with linezolid can increase the risk of severe liver failure and lactic acidosis, and liver biopsy may reveal vesicular steatosis. These risks are due to the effect of linezolid on mitochondria.

\section{Special notes}

(I) Old age, pre-existing liver disease, concurrent administration of other drugs, and excessive alcohol consumption can increase the risk of drug-mediated liver injury, although whether these factors may increase the hepatotoxicity of antibiotics remains unclear. In addition, although old age has been regarded as a risk factor, children and adolescents can also be affected by the same drug. The concurrent administration of acetaminophen (paracetamol) may affect the activity of the drug. The accompanying infection (in particular sepsis) can often lead to cholestasis.

(II) The hepatotoxicity of antibiotics is often seen after high-dose usage of these drugs; therefore, dose escalation of antibiotics (with an attempt to enhance efficacy) is associated with increased risk of hepatotoxicity.

(III) After an antibiotic is withdrawn due to the increased liver enzymes, the hepatotoxicity of the antibiotic may also disappear, although some other factors may also contribute to severe liver dysfunction.

(IV) Compared with the number of patients who have used antibiotics, cases with antibiotic-induced liver failure are rare.

\section{Antibiotic use in patients with renal insufficiency}

For patients with renal insufficiency, antibiotics with relatively low renal toxicity should be preferred. If an antibiotic with nephrotoxicity is required, a decreased dose or extended medication interval may be applied. The drug dose should be adjusted according to the degree of renal impairment to achieve tailored medication. For patients with renal insufficiency, the renal function should be evaluated thoroughly before establishing a medication regimen. The loading dose and maintenance dose should be determined; typically, the loading dose is used for the initial treatment, and then the dose is reduced. In kidney failure patients with severe infection, potent and effective antimicrobial treatment should be applied; during the first treatment, pulse dose is applied to rapidly achieve the effective drug concentration, followed by the use of maintenance dose $(35,36)$.

\section{Penicillins}

Penicillins are mainly secreted via kidneys, with rare nephrotoxicity. Generally, adjustment of dosage or extension of administration intervals is needed only when the glomerular filtration rate (GFR) is below $20 \mathrm{~mL} / \mathrm{min}$. In patients with renal failure, high-dose ampicillin can cause convulsions, hematopoietic function disorders and skin rashes, whereas penicillin $G$ and oxacillin occasionally can cause allergic interstitial nephritis. In patients with renal failure, penicillin in urine can maintain the effective antibiotic concentration; however, a large volume of penicillin in urine, as ions difficult to absorb, may lead to hypokalemic metabolic alkalosis. 


\section{Cephalosporins}

Many cephalosporins are mainly secreted via kidneys; in patients with renal insufficiency, their half-lives are remarkably extended and thus their doses need to be adjusted. Cefaloridine treatment may result in nephrotoxicity and should be avoided in patients with renal insufficiency.

\section{Aminoglycosides}

Aminoglycosides are highly nephrotoxic and thus should be used with caution in patients with renal insufficiency. If they are required for specific conditions, it is important to ensure sufficient kidney filling, strictly control the dosage, avoid the concurrent use with the cephalosporin antibiotics and/or diuretics, and avoid extended usage. Stop using aminoglycosides once the renal damage worsens.

\section{Chloramphenicol}

Chloramphenicol is mainly metabolized via the liver. However, its effect on bone marrow suppression remarkably increases in patients with uremia; in such circumstance, chloramphenicol should be used with caution.

\section{Erythromycin}

Erythromycin is mainly degraded via extra-renal route. Thus, its dose needs to be adjusted in patients with renal insufficiency.

\section{Tetracyclines}

Tetracyclines may aggravate uremia. If condition requires, a semisynthetic tetracycline (doxycycline or minocycline) that has been degraded outside the kidneys may be selected, without adjusting its dose.

\section{Quinolones}

As revealed by previous studies, for respiratory tract infection in patients with normal renal function to moderately renal insufficiency, garenoxacin $400 \mathrm{mg} / \mathrm{d}$ is appropriate; however, any dosage of garenoxacin is not feasible for patients with severe renal insufficiency (e.g., patients requiring hemodialysis). However, a recent research demonstrated that in patients with severe renal failure, garenoxacin $400 \mathrm{mg} / \mathrm{d}$ was effective for respiratory tract infections caused by common bacteria, and there was no severe adverse event (37).

Adjustment of the dosing regimen for patients with renal impairment

Reducing the dose or extending the administration intervals can maintain the blood drug concentration within a certain range, so as to avoid affecting the efficacy. No matter how the regimen is adjusted, the first dose should be administered using the normal dosage. Adjustment of the dosing regimen is based on the severity of renal impairment (mild, moderate, and severe); thus, the daily dose can be reduced to $2 / 3-1 / 2,1 / 2-1 / 5$, and $1 / 5-1 / 10$ of the normal dose; alternatively, the dose can also be adjusted according to blood drug concentration.

Table 1 shows the antibiotic dosage information (38).

\section{Part 5: main protocols of further studies in our current project}

\section{Solutions against MDR bacteria}

(I) Research on the new targets of antibiotics against MDR bacteria is particularly important.

(II) Combined antibiotic therapy remains the main solution against MDR bacteria. However, up to now few clinical studies have explored these combinations and their findings remain controversial; thus, multiple combinations should be tested to obtain the most effective antibiotic combination regimens against the MDR bacteria.

(III) Different administration routes should be investigated to achieve the highest drug concentrations at the infection sites.

(IV) Also, the proper antibiotic dosage and timing should be explored to avoid the non-effective use of antibiotics and lower the economic burden.

\section{Prophylaxis}

Although there is no official guidance on the specific perioperative antibiotic medications in the department of thoracic surgery (excluding the heart), the peri-operative prophylaxis for thoracic surgery should follow the Administrative Measures for the Clinical Use of Antibacterial Drugs [Order of the Ministry of Health (No. 84)] (the principles of prophylaxis for surgical operations).

\section{Prospect on anti-TB drugs and antifungal drugs}

Combination therapy remains the optimal treatment for MDR Mycobacterium tuberculosis infection. However, more clinical evidence is required due to the lack of clinical research. Meanwhile, R\&D of new drugs cannot be widely carried out 
Table 1 Adjustment of antibiotic dosage based on the changes in renal function

\begin{tabular}{|c|c|c|c|c|c|}
\hline \multirow{2}{*}{ Classes } & \multirow{2}{*}{ Antibiotics } & \multirow{2}{*}{$\begin{array}{l}\text { In patients with normal renal } \\
\text { function }\end{array}$} & \multicolumn{3}{|c|}{$\begin{array}{c}\begin{array}{c}\text { Dosage adjustment based on different GFR in patients with renal } \\
\text { disease }\end{array}\end{array}$} \\
\hline & & & $\begin{array}{c}\text { GFR }>50 \mathrm{~mL} / \mathrm{min} / \\
1.73 \mathrm{~m}^{2}\end{array}$ & $\begin{array}{c}\text { GFR } 10-50 \mathrm{~mL} / \mathrm{min} / \\
1.73 \mathrm{~m}^{2}\end{array}$ & $\begin{array}{c}\text { GFR }<10 \mathrm{~mL} / \mathrm{min} / \\
1.73 \mathrm{~m}^{2}\end{array}$ \\
\hline \multirow[t]{13}{*}{ Cephalosporins } & Cefixime & $250 \mathrm{mg}$, bid & $100 \%$ & $75 \%$ & $50 \%$ \\
\hline & Cefotaxime & $1 \mathrm{~g}$, qid & Qid & Bid or tid & Qd \\
\hline & Cefoxitin & $1-2 \mathrm{~g}$, tid or qid & Tid & Bid or tid & $Q(1-2) d$ \\
\hline & Cefpodoxime & 200 mg, bid & Bid & Q16h & $Q(1-2) d$ \\
\hline & Cefprozil & $500 \mathrm{mg}$, bid & $250 \mathrm{mg}$, bid & $250 \mathrm{mg}, \mathrm{q}(12-16) \mathrm{h}$ & $250 \mathrm{mg}, \mathrm{qd}$ \\
\hline & Ceftazidime & $1-2 \mathrm{~g}$, tid & Bid or tid & $\mathrm{Q}(1-2) \mathrm{d}$ & Q2d \\
\hline & Cefuroxime sodium & $0.75-1.50 \mathrm{~g}, \mathrm{tid}$ & Tid & Bid or tid & Bid \\
\hline & Cefalexin & 250-500 mg, qid & Tid & Bid & $Q(1-2) d$ \\
\hline & Cefepime & $0.25-2.00 \mathrm{~g}, \mathrm{tid}$ & Bid & $\mathrm{Q}(16-24) \mathrm{h}$ & $50 \%$, qd \\
\hline & Cefditoren & 200-400 mg, bid & $100 \%$ & $50 \%$ & $50 \%$, qd \\
\hline & Cefdinir & 300 mg, bid & Bid & Bid & Qd \\
\hline & Cefazolin & $0.5-2.0 \mathrm{~g}$, tid & Tid & Bid & $Q(1-2) d$ \\
\hline & Cefadroxil & $0.5-1.0 \mathrm{~g}$, bid & Bid & Qd or bid & $Q(1-2) d$ \\
\hline Aminoglycosides & Tobramycin & $1.0-1.7 \mathrm{mg} / \mathrm{kg}$, tid & $60-90 \%$, bid or tid & $30-70 \%$, bid & $20-30 \%, q(1-2) d$ \\
\hline \multirow[t]{3}{*}{ Carbapenem } & Ertapenem & $1 \mathrm{~g}, \mathrm{qd}$ & $100 \%$ & $100 \%$ & $50 \%$ \\
\hline & Imipenem & $0.25-1.00 \mathrm{~g}$, qid & $100 \%$ & $50 \%$ & $25 \%$ \\
\hline & Meropenem & $0.5-1.0 \mathrm{~g}$, qid & $0.5 \mathrm{~g}$, qid & $0.25-0.50 \mathrm{~g}, \mathrm{bid}$ & $0.25-0.5 \mathrm{~g}, \mathrm{qd}$ \\
\hline \multirow[t]{2}{*}{ Macrolides } & Clarithromycin & 500 mg, bid & $100 \%$ & $75 \%$ & $50-75 \%$ \\
\hline & Erythromycin & $250-500 \mathrm{mg}, \mathrm{bid}, \mathrm{tid}$, or qid & $100 \%$ & $100 \%$ & $50-75 \%$ \\
\hline \multirow[t]{5}{*}{ Penicillins } & Amoxicillin & 250-500 mg, tid & Tid & Bid or tid & Qd \\
\hline & Ampicillin & $250 \mathrm{mg}-2 \mathrm{~g}$, qid & Qid & Bid, tid, or qid & Qd or bid \\
\hline & Penicillin G & $500 \mathrm{k}-4 \mathrm{~m} \mathrm{IU}, \mathrm{q} 4 \mathrm{~h}$ & $100 \%$ & $75 \%$ & $50 \%$ \\
\hline & Piperacillin & $3-4 \mathrm{~g}, \mathrm{q} 4 \mathrm{~h}$ & Qid-q4h & Tid or qid & Tid \\
\hline & Ticarcillin & $3 \mathrm{~g}, \mathrm{q} 4 \mathrm{~h}$ & $1-2 \mathrm{~g}, \mathrm{q} 4 \mathrm{~h}$ & $1-2 \mathrm{~g}$, tid & $1-2 \mathrm{~g}$, bid \\
\hline
\end{tabular}

Table 1 (continued) 
Table 1 (continued)

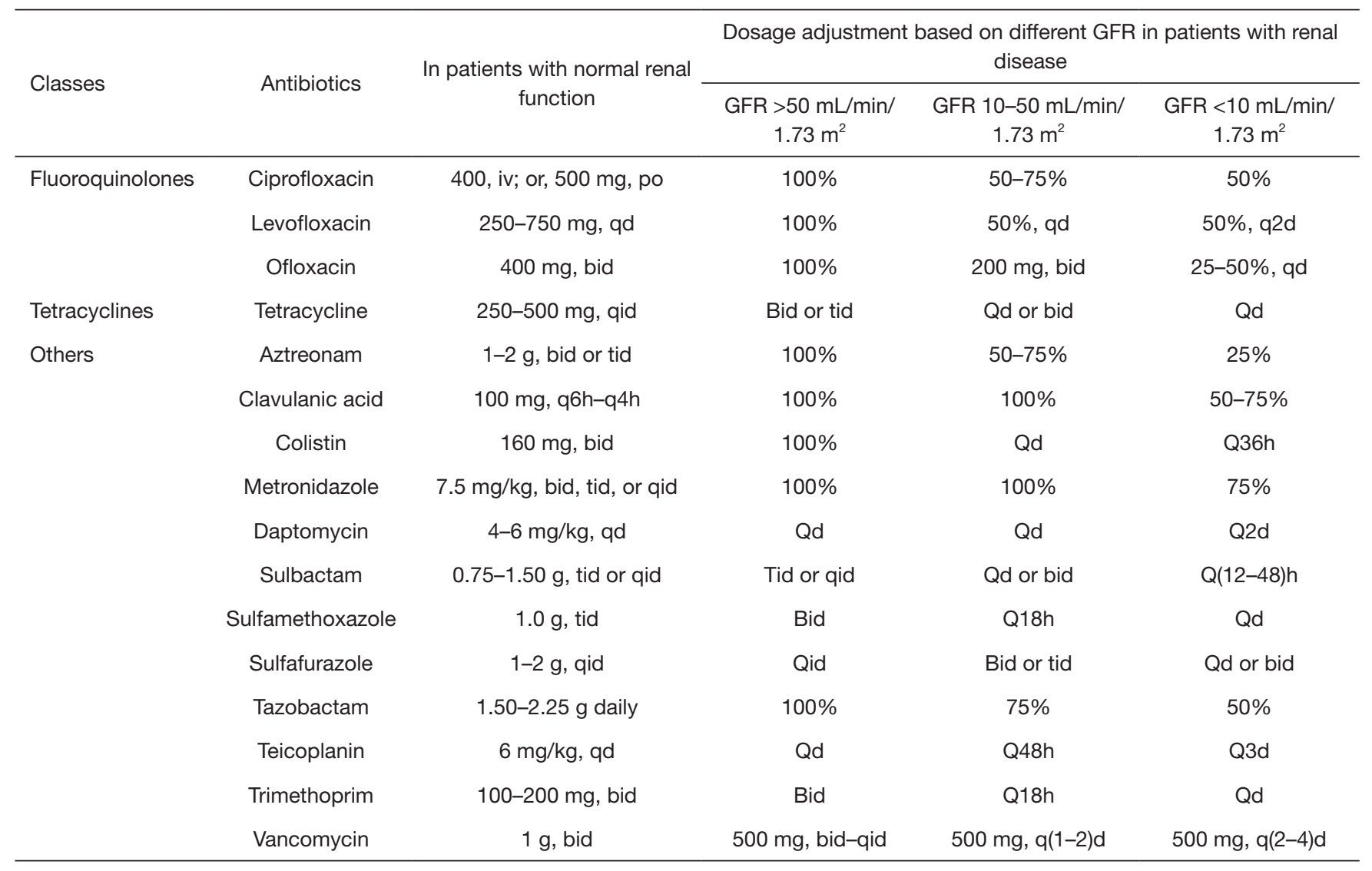

Some synthetic antibacterial drugs and antifungal drugs that can be clinically used in patients with renal insufficiency are also included in this table. GFR, glomerular filtration rate.

due to the small number of clinical trials and the increased risk of death. Therefore, adequate clinical trials are warranted.

Fungal infections often occur in immunosuppressed patients, especially in patients with chemotherapy-induced neutropenia or in patients who are using glucocorticoids for GVHD. In patients with neutropenia, the recovery of bone marrow function is critical for the control of aspergillosis. Under such circumstances, lowering the level of immune suppression shall be used as an adjunct to anti-fungal therapy. Study on how to reduce the degree of immune suppression may be a way of improving clinical outcomes.

\section{Antibiotic use in patients with poor bepatic/renal function}

(I) Monitor the blood drug concentration;

(II) Strictly follow the principles of antibiotic use; and

(III) Strengthen clinical trials and pharmacokinetic studies, so as to optimize antibiotic use and minimize side effect on renal function.

\section{Acknowledgements}

None.

\section{Footnote}

Conflicts of Interest: The authors have no conflicts of interest to declare.

\section{References}

1. Devasahayam G, Scheld WM, Hoffman PS. Newer antibacterial drugs for a new century. Expert Opin Investig Drugs 2010;19:215-34.

2. Otter JA, French GL. Molecular epidemiology of community-associated meticillin-resistant Staphylococcus 
aureus in Europe. Lancet Infect Dis 2010;10:227-39.

3. Burgess DS, Rapp RP. Bugs versus drugs: addressing the pharmacist's challenge. Am J Health Syst Pharm 2008;65:S4-15.

4. Experts on Rational Use of Drugs, National Health and Family Planning Commission. National Drug Resistance Report 2015 of China, 2015.

5. Zhang $\mathrm{H}, \mathrm{Xu}$ YC. Analysis on pathogen distribution and drug sensibility in Peking Union Medical College Hospital 2015. 2015.

6. Zhang B, Hu Y, Zhao J, et al. Antimicrobial utilization and antimicrobial resistance trend before and after implementation of a 3-year national antimicrobial stewardship program in a tertiary hospital. Chinese Pharmaceutical Journal 2014;49:2136-40.

7. Kumarasamy KK, Toleman MA, Walsh TR, et al. Emergence of a new antibiotic resistance mechanism in India, Pakistan, and the UK: a molecular, biological, and epidemiological study. Lancet Infect Dis 2010;10:597-602.

8. Guidelines on the Clinical Application of of Antibacterial Drugs. Order of Ministry of Health (No. 285), 2049.

9. Administrative Measures for the Clinical Use of Antibacterial Drugs. Order of the Ministry of Health (No. 84).

10. Notice of the Office of the Ministry of Health on the Administration of the Clinical Application of Antibacterial Drugs. Order of Ministry of Health (No. 38), 2009.

11. Papp-Wallace KM, Endimiani A, Taracila MA, et al. Carbapenems: past, present, and future. Antimicrob Agents Chemother 2011;55:4943-60.

12. Roberts JA, Kirkpatrick CM, Roberts MS, et al. Meropenem dosing in critically ill patients with sepsis and without renal dysfunction: intermittent bolus versus continuous administration? Monte Carlo dosing simulations and subcutaneous tissue distribution. J Antimicrob Chemother 2009;64:142-50.

13. Lorente L, Lorenzo L, Martín MM, et al. Meropenem by continuous versus intermittent infusion in ventilatorassociated pneumonia due to gram-negative bacilli. Ann Pharmacother 2006;40:219-23.

14. Ceccarelli G, Falcone M, Giordano A, et al. Successful ertapenem-doripenem combination treatment of bacteremic ventilator-associated pneumonia due to olistin-resistant KPC-producing Klebsiella pneumoniae. Antimicrob Agents Chemother 2013;57:2900-1.

15. Li J, Nation RL, Milne RW, et al. Evaluation of colistin as an agent against multi-resistant Gram-negative bacteria. Int J Antimicrob Agents 2005;25:11-25.
16. Durante-Mangoni E, Signoriello G, Andini R, et al. Colistin and rifampicin compared with colistin alone for the treatment of serious infections due to extensively drug-resistant Acinetobacter baumannii: a multicenter, randomized clinical trial. Clin Infect Dis 2013;57:349-58.

17. Aydemir H, Akduman D, Piskin N, et al. Colistin vs. the combination of colistin and rifampicin for the treatment of carbapenem-resistant cinetobacter baumannii ventilator-associated pneumonia. Epidemiol Infect 2013;141:1214-22.

18. Garnacho-Montero J, Amaya-Villar R, Gutiérrez-Pizarraya A, et al. Clinical efficacy and safety of the combination of colistin plus vancomycin for the treatment of severe infections caused by carbapenem-resistant A baumannii. Chemotherapy 2013;59:225-31.

19. Petrosillo N, Giannella M, Antonelli M, et al. Colistinglycopeptide combination in critically ill patients with Gram negative infection: the clinical experience. Antimicrob Agents Chemother 2014;58:851-8.

20. Arroyo LA, Mateos I, González V, et al. In vitro activities of tigecycline, minocycline, and colistin-tigecycline combination against multi- and pandrug-resistant clinical isolates of Acinetobacter baumannii group. Antimicrob Agents Chemother 2009;53:1295-6.

21. Ramirez J, Dartois N, Gandjini H, et al. Randomized phase 2 trial to evaluate the clinical efficacy of two highdosage tigecycline regimens versus imipenem-cilastatin for treatment of hospitalacquired pneumonia. Antimicrob Agents Chemother 2013;57:1756-62.

22. Mimoz O, Rolland D, Adoun M, et al. Steady-state trough serum and epithelial lining fluid concentrations of teicoplanin $12 \mathrm{mg} / \mathrm{kg}$ per day in patients with ventilatorassociated pneumonia. Intensive Care Med 2006;32:775-9.

23. Kollef MH, Rello J, Cammarata SK, et al. Clinical cure and survival in Gram-positive ventilator-associated pneumonia: retrospective analysis of two double-blind studies comparing linezolid with vancomycin. Intensive Care Med 2004;30:388-94.

24. Wunderink RG, Niederman MS, Kollef MH, et al. Linezolid in methicillin-resistant Staphylococcus aureus nosocomial pneumonia: a randomized, controlled study. Clin Infect Dis 2012;54:621-9.

25. Freire AT, Melnyk V, Kim MJ, et al. Comparison of tigecycline with imipenem/cilastatin for the treatment of hospital-acquired pneumonia. Diagn Microbiol Infect Dis 2010;68:140-51.

26. Michalopoulos A, Fotakis D, Virtzili S, et al. Aerosolized colistin as adjunctive treatment of ventilator-associated 
pneumonia due to multidrug-resistant Gram-negative bacteria: a prospective study. Respir Med 2008;102:407-12.

27. Chastre J, Wolff M, Fagon JY, et al. Comparison of 8 vs. 15 days of antibiotic therapy for ventilatorassociated pneumonia in adults: a randomized trial. JAMA 2003;290:2588-98.

28. Dimopoulos G, Poulakou G, Pneumatikos IA, et al. Shortvs. long-duration antibiotic regimens for ventilatorassociated pneumonia: a systematic review and metaanalysis. Chest 2013;144:1759-67.

29. Stolz D, Smyrnios N, Eggimann P, et al. Procalcitonin for reduced antibiotic exposure in ventilator-associated pneumonia: a randomised study. Eur Respir J 2009;34:1364-75.

30. Diacon AH, Pym A, Grobusch M, et al. The diarylquinoline TMC207 for multidrug-resistant tuberculosis. N Engl J Med 2009;360:2397.

31. Diacon AH, Donald PR, Pym A, et al. Randomized pilot trial of eight weeks of bedaquiline (TMC207) treatment for multidrug-resistant tuberculosis: long-term outcome, tolerability, and effect on emergence of drug resistance. Antimicrob Agents Chemother 2012;56:3271.

Cite this article as: Li S, Han Z, He J, Gao S, Liu D, Liu L, He J, Huang Y, Xu S, Mao W, Tan Q, Chen C, Li X, Zhang Z, Jiang G, Xu L, Zhang L, Fu J, Li H, Wang Q, Tan L, Li D, Zhou Q, Fu X, Jiang Z, Chen H, Fang W, Zhang X, Li Y, Tong T, Yu Z, Liu Y, Zhi X, Yan T, Zhang X, Imperatori A, Ibrahim M, Novoa NM, Ng CS, Petersen RH, Chen JS, Fukuchi Y, Brunelli A, Ismail M, Valverde JA, Rodriguez-Lucas C. Society for Translational Medicine expert consensus on the use of antibacterial drugs in thoracic surgery. J Thorac Dis 2018;10(11):6356-6374. doi: 10.21037/jtd.2018.10.108
32. Diacon AH, Pym A, Grobusch MP, et al. Multidrugresistant tuberculosis and culture conversion with bedaquiline. N Engl J Med 2014;371:723.

33. Vazquez JA. Clinical practice: combination antifungal therapy for mold infections: much ado about nothing? Clin Infect Dis 2008;46:1889-901.

34. Krishna G, Martinho M, Chandrasekar P, et al. Pharmacokinetics of oral posaconazole in allogeneic hematopoietic stem cell transplant recipients with graftversus-host disease. Pharmacotherapy 2007;27:1627.

35. Lou WJ, He LJ, Wang HM. Rational application of antibiotics in patients with renal insufficiency. World Clinic Drugs 2012;33.

36. Wang CX, Mei Y. Antibiotic use in patients with renal insufficiency in the surgical department. Chinese Journal of General Surgery 1995;(1):20-2.

37. Yamagishi Y, Hagihara M, Hamada Y, et al. Pharmacokinetic study of garenoxacin in severe renal failure patients. Jpn J Antibiot 2015;68:141-50.

38. Ye RG, Yu XQ. Rational use of antibiotics in patients with renal failure. Chinese Journal of Practical Surgery 1991. 